\title{
Influencing Factors of Successful Eradication of Multidrug-Resistant Acinetobacter baumannii in the Respiratory Tract with Aerosolized Colistin
}

\author{
Yu-Mu Chen ${ }^{1 *}$, Wen-Feng Fang ${ }^{1,2,3 *}$, Hsu Ching Kao ${ }^{1}$, Hung-Chen Chen ${ }^{1}$, Yuh-Chyn Tsai ${ }^{3}$, \\ Lien-Shi Shen ${ }^{3}$, Chin-Ling Li ${ }^{3}$, Huang-Chih Chang ${ }^{1}$, Kuo-Tung Huang ${ }^{1}$, Meng-Chih Lin ${ }^{1,2,3}$, \\ Chin-Chou Wang ${ }^{1,2,3,4}$, Tung-Ying $\mathrm{Chao}^{1}$
}

Background: The increased prevalence of multidrug-resistant Acinetobacter baumannii (MDRAB) poses a worldwide treatment challenge. Although aerosolized colistin therapy for MDRAB pneumonia has attracted increasing interest, factors influencing successful eradication remain unclear. Methods: This retrospective study evaluated 135 consecutively admitted adult patients showing positive respiratory secretion cultures for MDRAB who underwent aerosolized colistin therapy between January 2007 and November 2011. Possible factors related to pneumonia and MDRAB eradication were collected for analysis.

Results: $\quad$ Patients with successful MDRAB eradication on Day 14 had a shorter interval between the day the positive MDRAB sputum cultures were yielded and the day colistin inhalation treatment began $(4.0 \pm 2.5$ vs. $7.3 \pm 6.5 ; p=0.002)$. Patients with a worsening chest X-ray on Day 7 of the colistin inhalation had a lower chance of 14-day MDRAB eradication [1/44 (2.3\%) vs. 8/37 (21.6\%); $p=0.006]$. Patients with diabetes mellitus also had a lower chance of early MDRAB eradication [13/44 (29.5\%) vs. 20/37 (54.1\%); $p=0.025]$.

\begin{abstract}
At a Glance Commentary
Scientific background of the subject

Multidrug-resistant Acinetobacter baumannii (MDRAB) poses a worldwide challenge for treatment and infection control. Factors predicting successful eradication using aerosolized colistin remain unclear.

\section{What this study adds to the field}

Early intervention using aerosolized colistin in patients with MDRAB pneumonia or colonization can achieve better eradication. However, patients with diabetes mellitus had lower 14-day eradication rate and patients with worsening CXR on Day 7 of the colistin inhalation had a lower chance of early MDRAB eradication.
\end{abstract}

Conclusions: Early intervention using aerosolized colistin in patients with MDRAB pneumonia or colonization can achieve better eradication.

(Biomed J 2014;37:269-283)

\section{Key words: acinetobacter baumannii, aerosolized colistin, multidrug resistant}

$\mathrm{T}_{b}^{\mathrm{h}}$ The increased prevalence of multidrug-resistant Acinetobacter baumannii (MDRAB) poses a worldwide challenge for treatment and infection control. ${ }^{[1-3]}$ MDRAB can colonize in the respiratory tracts or lead to nosocomial pneumonia. ${ }^{[4]}$
MDRAB pneumonia has high mortality rates, and leads to increased hospital stay lengths and costs. ${ }^{[5,6]}$

Colistin, a polypeptide antibiotic available since the 1960s, has rarely been used because of a high incidence of

\footnotetext{
*The authors contributed equally to this work.

From the ${ }^{1}$ Division of Pulmonary and Critical Care Medicine, Department of Internal Medicine, Kaohsiung Chang Gung Memorial Hospital and Chang Gung University College of Medicine, Kaohsiung, Taiwan; ${ }^{2}$ Department of Respiratory Care, Chang Gung University of Science and Technology, Chiayi, Taiwan; ${ }^{3}$ Department of Respiratory Therapy, Kaohsiung Chang Gung Memorial Hospital and Chang Gung University College of Medicine, Kaohsiung, Taiwan; ${ }^{4}$ Graduate Institute of Occupational Safety and Health, Kaohsiung Medical University, Kaohsiung, Taiwan Received: Feb. 22, 2013; Accepted: Oct. 02, 2013

Correspondence to: Dr. Tung-Ying Chao, Division of Pulmonary and Critical Care Medicine, Department of Internal Medicine, Kaohsiung Chang Gung Memorial Hospital. 123, Dapi Rd., Niaosong, Kaohsiung 833, Taiwan (ROC). Tel: 886-7-7317123 ext. 8199; Fax: 886-7-7322402; E-mail: tychao@adm.cgmh.org.tw

Correspondence to: Dr. Chin-Chou Wang, Division of Pulmonary and Critical Care Medicine, Department of Internal Medicine, Kaohsiung Chang Gung Memorial Hospital. 123, Dapi Rd., Niaosong, Kaohsiung 833, Taiwan (ROC). Tel: 886-7-7317123 ext. 8199; Fax: 886-7-7322402; E-mail: ccwang5202@yahoo.com.tw
}

DOI: $10.4103 / 2319-4170.132879$ 
nephrotoxicity reported in previous clinical data ${ }^{[7,8]}$ Its usage has been restricted to the treatment and prevention of recurrent Pseudomonas aeruginosa pneumonia in patients with cystic fibrosis. ${ }^{[9,10]}$ However, increasing rates of resistance among Gram-negative bacteria to current empiric antibiotics and a subsequent high clinical failure rate has caused colistin to be re-evaluated for MDRAB infection treatment. ${ }^{[11-14]}$ Although intravenous (IV) colistin administration against MDRAB may yield high clinical and microbial success rates, ${ }^{[15]}$ recent studies have reported a nephrotoxicity rate of $10-30 \% .^{[16,17]}$ Use of aerosolized colistin to treat MDRAB pneumonia or to eradicate the pathogen from the respiratory tract has been tested. ${ }^{[11-13,18]}$ However, factors influencing successful eradication remain unclear.

In this study, we attempted to determine the factors influencing successful MDRAB eradication using aerosolized colistin.

\section{METHODS}

\section{Patient population}

This retrospective study was conducted at a 2500-bedded tertiary teaching hospital in southern Taiwan. According to our hospital guidelines, patients with a positive sputum culture for MDRAB are required to undergo contact isolation until two sets of successive sputum cultures yield negative results for MDRAB growth. We assessed all adult patients (age $\geq 18$ years) who were admitted in the respiratory care center from January 2007 to November 2011 with at least one set of cultures from respiratory secretions (including sputum, trachea suction, and bronchial washing) that yielded MDRAB microbial growth. Patients were enrolled if they had received aerosolized (inhaled) colistin for more than 3 days after the day sputum culture yielded positive MDRAB growth. Control group of patients received other antibiotic therapies that did not include inhaled colistin or were given no antibiotic at all. Patients were excluded if they had concomitant MDRAB bacteremia because it required an IV antibiotic (instead of only aerosolized therapy), and no such patient received aerosolized (inhaled) colistin during our study period. Patients who died or were discharged within 14 days after the discovery of a positive MDRAB culture were excluded. To limit the confounding factors, patients who received IV colistin with or without aerosolized colistin were also excluded. The study was approved by the Institutional Review Board of Chang Gung Memorial Hospital (permit number of IRB: 100-4062B), and the patient consent requirement was waived.

\section{Colistin regimen}

The aerosolized colistin dosage was 2 million international units (IU) (or $160 \mathrm{mg}$ colistin methanesulfonate) $)^{[11]}$ administered twice per day. No dose adjustment was made based on the patients' renal function. In spontaneously breathing patients, 2 million IU colistin was diluted in $4 \mathrm{ml}$ normal saline and nebulized with 8 1/min oxygen flow. For patients with ventilator support, the aerosolized colistin was diluted in $2 \mathrm{ml}$ of sterile normal saline $(0.9 \%)$ and delivered via a ventilator.

\section{Data collection and definition}

Clinical data, including baseline patient characteristics, possible predisposing factors for MDRAB infection, bacteriological and clinical responses of MDRAB infection/ colonization, and patient outcomes were retrospectively collected using a review of medical records. We compared the changes of the chest X-ray (CXR) and systemic inflammatory response syndrome (SIRS) scores between the first day of colistin inhalation treatment and $7( \pm 2)$ days later. The interval between the day that the sputum culture yielded MDRAB and the day that the aerosolized colistin therapy began was also recorded.

\section{Definition of multidrug resistance}

Multidrug resistance was defined as isolates that were insusceptible to all of the following antibiotics: Anti-pseudomonal penicillins, anti-pseudomonal cephalosporins, carbapenems, monobactams, quinolones, aminoglycosides, tetracycline, and trimethoprim/sulfamethoxazole. ${ }^{[14,19]}$

Antimicrobial susceptibility of $A$. baumannii isolates was determined using the disk-diffusion test, as recommended by the National Committee for Clinical Standards.

\section{Definition of colonization/pneumonia}

Pneumonia was diagnosed based on the US Centers for Disease Control and Prevention criteria, with at least two or more of the following to be satisfied: (1) fever (body temperature $>38.3^{\circ} \mathrm{C}$ ); (2) leukocytosis $(25 \%$ increase and $>10000 / \mathrm{mm}^{3}$ ) or leukopenia (25\% decrease and $<5000 / \mathrm{mm}^{3}$ ); and (3) purulent tracheal secretion. One of the following criteria must also be satisfied: (1) new and persistent infiltrates appearing on the chest radiograph, (2) the same microorganisms are isolated from pleural fluid and tracheal secretions, (3) a radiographic cavitation, (4) histological proof of pneumonia, or (5) positive cultures from the bronchoalveolar lavage $\left(\geq 1 \times 10^{4}\right.$ colony-forming units/ml). ${ }^{[14,18]}$

The CXR and its change was defined as infiltration, consolidation, cavitations; improvement, stationary, or progression by one radiologist and one pulmonologist. If they had contradicting opinions, another senior pulmonologist was consulted. If the patients' clinical presentations did not meet the criteria for pneumonia, they were classified as colonization. 


\section{Definition of 14- or 28-day eradication}

Sputum cultures were performed every 3-5 days from the day the sputum yielded positive growth for MDRAB. The 14-day eradication was defined as a minimum of two consecutive cultures from the respiratory secretions that revealed no MDRAB growth within 14 days. ${ }^{[11]}$ The 28-day eradication was defined as at least two consecutive cultures from the respiratory secretions that yielded no MDRAB growth within 28 days. ${ }^{[11]}$

\section{Definition of SIRS score}

The SIRS score is a four-point score defined in this study, marking one point for each of the four components: (1) body temperature less than $36^{\circ} \mathrm{C}$ or greater than $38^{\circ} \mathrm{C}$, (2) heart rate greater than 90 beats/min, (3) tachypnea (high respiratory rate) with a breathing rate greater than 20 breaths/min or an arterial partial pressure of carbon dioxide less than $32 \mathrm{~mm} \mathrm{Hg}$, (4) white blood cell count less than 4000 cells $/ \mathrm{mm}^{3}\left(4 \times 10^{9}\right.$ cells/l) or greater than 12,000 cells $/ \mathrm{mm}^{3}\left(12 \times 10^{9}\right.$ cells $\left./ 1\right)$, or the presence of greater than $10 \%$ of immature neutrophils (band forms). ${ }^{[20]}$

\section{Statistical analysis}

Categorical variables were analyzed using the Chi-square test or Fisher's exact test where appropriate, and continuous variables were compared using Student's $t$-test or the Mann-Whitney U test. Multivariate logistic regression analysis was performed to identify the factors for early MDRAB eradication. All variables that were considered risk factors with $p<0.10$ in the univariate analysis were entered into the multivariate model. If individual variables associated with early MDRAB eradication had $p<0.05$ in the multivariate model, a backward elimination procedure was used to identify the final independent factors. Adjusted odds ratios (AORs) and 95\% confidence intervals (CIs) were reported for logistic regression analysis. A two-tailed $p<0.05$ was considered significant. All statistical analyses were performed using the SPSS 18.0 software package (SPSS Inc., Chicago, IL, USA).

\section{RESULTS}

A total of 240 patients with MDRAB growth from respiratory secretions were admitted to Kaohsiung Chang Gung Memorial Hospital from January 2007 to November 2011. Seventy-seven patients were excluded because concomitant MDRAB bacteremia was identified and those patients did not receive aerosolized colistin therapy. Another 28 patients were excluded because of concomitant use of IV colistin. The baseline characteristics for all 135 patients enrolled are shown in Table 1 . There was no statistical difference between the aerosolized colistin group $(n=81)$ and the control group $(n=54)$ regarding sex, age, severity score [Acute Physiological and Chronic Health Evaluation II (APACHE II)], and underlying comorbidities.

Fifty-two patients were diagnosed with MDRAB pneumonia, and 83 patients were diagnosed with MDRAB colonization. In addition, 27 patients in the aerosolized group received a concomitant IV antibiotic therapy (22 with Tigecycline and five with Unasyn).

The most commonly prescribed antibiotic for the control group patients was Tigecycline $(n=40)$, followed by Unasyn $(n=12)$. Two other patients did not receive antibiotic treatment, despite showing respiratory tract colonization with MDRAB.

Physicians preferred using aerosolized colistin compared to other IV antibiotics to eradicate MDRAB colonization $(70.4 \%$ vs. $48.1 \% ; p=0.009)$. Physicians preferred using IV antibiotic, rather than aerosolized colistin, to treat MDRAB-induced pneumonia (51.9\% vs. 29.6\%; $p=0.009)$.

\section{Outcomes between aerosolized group and control group}

The early MDRAB eradication rate within 14 days after the first observation of MDRAB isolation from the respiratory secretions was higher in patients treated with aerosolized colistin than in patients treated with other medication [54.3\% (44/81) vs. 29.6\% (16/54); $p=0.005$ ] [Table 2].

No difference was seen between the aerosolized colistin group and the control group patients for the respiratory tract MDRAB eradication rate on Day 28 [66.7\% (54/81)

Table 1: Baseline patient characteristics

\begin{tabular}{lccc}
\hline Variable & $\begin{array}{c}\text { Aerosolized } \\
\text { colistin } n=81(\%)\end{array}$ & $\begin{array}{c}\text { Control } \\
n=54(\%)\end{array}$ & $p$ value \\
\hline Age, mean years \pm SD & $74.9 \pm 12.0$ & $71.2 \pm 15.7$ & 0.190 \\
Male & $50(61.7)$ & $27(50.0)$ & 0.177 \\
Apache II score*, mean \pm SD & $19.8 \pm 5.5$ & $22.5 \pm 5.4$ & 0.171 \\
Colonization & $57(70.4)$ & $26(48.1)$ & 0.009 \\
Pneumonia & $24(29.6)$ & $28(51.9)$ & 0.009 \\
Smoking & $14(17.3)$ & $8(14.8)$ & 0.704 \\
Alcoholism & $6(7.4)$ & $4(7.4)$ & 1.000 \\
Diabetes mellitus & $33(40.7)$ & $15(27.8)$ & 0.123 \\
Hypertension & $49(60.5)$ & $27(50.0)$ & 0.229 \\
Coronary artery disease & $19(23.5)$ & $9(16.7)$ & 0.340 \\
Chronic kidney disease & $16(19.8)$ & $13(24.1)$ & 0.549 \\
Liver cirrhosis & $3(3.8)$ & $2(3.7)$ & 0.989 \\
COPD & $18(22.2)$ & $6(11.1)$ & 0.098 \\
Neoplastic disease & $15(18.5)$ & $10(18.5)$ & 0.714 \\
Stroke & $24(29.6)$ & $11(20.4)$ & 0.229 \\
Tuberculosis history & $7(8.6)$ & $4(7.4)$ & 0.797 \\
Tracheostomy & $6(7.4)$ & $6(11.1)$ & 0.689
\end{tabular}

Abbreviations: SD: Standard deviation; APACHE: Acute physiology and chronic health evaluation; COPD: Chronic obstructive pulmonary disease; *: Apache II score was measured at days admitted to intensive care unit or respiratory care center 
vs. $51.9 \%$ (28/54); $p=0.084]$. In addition, there was no statistical difference in the 28-day mortality rate between patients treated with aerosolized colistin and the control group [11.3\% (6/81) vs. $16.7 \%(8 / 54) ; p=0.167]$.

In addition, no statistical difference in hospital mortality between patient groups was apparent [32.1\% (26/81) vs. $40.7 \%$ (22/54); $p=0.304]$.

\section{Predictors for early MDRAB eradication with aerosolized colistin}

Sex, age, severity score (APACHE II), and tracheostomy status did not alter the eradication rate on Day 14 [Table 3]. However, analyzing the influence of underlying comorbidities on the 14-day MDRAB eradication showed that patients with diabetes mellitus (DM) had lower 14-day eradication rate [13/44 (29.5\%) vs. 20/37 (54.1\%); $p=0.025]$.

Patients with successful MDRAB eradication on Day 14 had a shorter interval between the day the sputum cultures yielded MDRAB positive growth and the day colistin inhalation treatment began $(4.0 \pm 2.5$ vs. $7.3 \pm 6.5$;

Table 2: Outcomes of patients in aerosolized colistin group and control group

\begin{tabular}{lccc}
\hline Variable & $\begin{array}{c}\text { Aerosolized colistin } \\
n=81(\%)\end{array}$ & $\begin{array}{c}\text { Control } \\
n=54(\%)\end{array}$ & $p$ value \\
\hline 14-day eradication & $44(54.3)$ & $16(29.6)$ & 0.005 \\
28-day eradication & $54(66.7)$ & $28(51.9)$ & 0.084 \\
28-day mortality & $6(7.4)$ & $8(14.8)$ & 0.167 \\
In-hospital mortality & $26(32.1)$ & $22(40.7)$ & 0.304 \\
$\begin{array}{l}\text { Duration of hospital stay, } \\
\text { mean } \pm \text { SD }\end{array}$ & $67.4 \pm 44.0$ & $62.3 \pm 52.6$ & 0.541 \\
\hline
\end{tabular}

Abbreviation: SD: Standard deviation $p=0.002$ ) [Figure 1]. In addition, the status of the pneumonia or respiratory tract colonization with MDRAB did not affect the 14-day eradication rate. However, the CXR resolution on Day 7 cannot be used to determine whether the MDRAB in the respiratory tract can be eradicated on Day 14, and patients with a worsening CXR on Day 7 of the colistin inhalation had a lower chance of early MDRAB eradication [1/44 (2.3\%) vs. 8/37 (21.6\%); $p=0.006]$ [Table 4]. The same result was also found with regard to SIRS score; improved score on Day 7 cannot be used to determine whether the MDRAB in the respiratory tract can be eradicated on Day 14, and patients with a worsening SIRS score on Day 7 of the colistin inhalation had a borderline

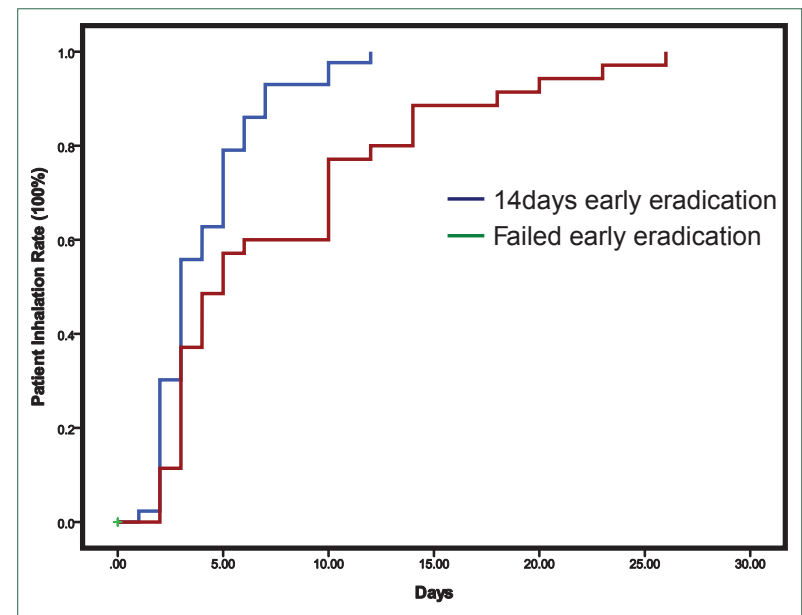

Figure 1: Cumulative percentage of patients started on inhalation colistin after the sputum culture yielded multidrug-resistant Acinetobacter baumannii between successful and failed 14-day eradication groups.

Table 3: The influence of baseline characteristics on MDRAB eradication on day 14

\begin{tabular}{|c|c|c|c|c|c|}
\hline Variable & $\begin{array}{c}\text { 14-day eradication } \\
n=44(\%)\end{array}$ & $\begin{array}{c}\text { Failed 14-day eradication } \\
n=37(\%)\end{array}$ & $\begin{array}{c}\text { Univariate analysis } \\
\quad p \text { value }\end{array}$ & Odds ratio $(95 \% \mathrm{CI})$ & $\begin{array}{l}\text { Multivariate analysis } \\
\quad p \text { value }\end{array}$ \\
\hline Age, mean years $\pm \mathrm{SD}^{*}$ & $74.7 \pm 12.9$ & $74.0 \pm 10.9$ & 0.808 & & \\
\hline Male & $28(63.6)$ & $22(59.5)$ & 0.700 & $1.2(0.5-2.9)$ & \\
\hline Apache II score $\dagger$, mean \pm SD* & $24.0 \pm 5.9$ & $23.5 \pm 5.0$ & 0.697 & & \\
\hline Smoking & $10(22.7)$ & $4(10.8)$ & 0.158 & $2.4(0.7-8.5)$ & \\
\hline Alcoholism & $3(6.8)$ & $3(8.1)$ & 0.825 & $0.8(0.2-4.4)$ & \\
\hline Diabetes mellitus & $13(29.5)$ & $20(54.1)$ & 0.025 & $0.4(0.1-0.9)$ & 0.041 \\
\hline Hypertension & $27(61.4)$ & $22(59.5)$ & 0.861 & $1.1(0.4-2.6)$ & \\
\hline Coronary artery disease & $11(25.0)$ & $8(21.6)$ & 0.721 & $1.2(0.4-3.4)$ & \\
\hline Chronic kidney disease & $6(13.6)$ & $10(27.0)$ & 0.132 & $0.4(0.1-1.3)$ & \\
\hline Liver cirrhosis & $0(0)$ & $3(8.3)$ & 0.087 & $0(0-0)$ & 0.999 \\
\hline $\mathrm{COPD} \ddagger$ & $11(25.0)$ & $7(18.9)$ & 0.512 & $1.4(0.5-4.1)$ & \\
\hline Neoplastic disease & $10(22.7)$ & $5(13.5)$ & 0.351 & $1.9(0.6-6.1)$ & \\
\hline Stroke & $11(25.0)$ & $13(35.1)$ & 0.320 & $0.6(0.2-1.6)$ & \\
\hline Tuberculosis history & $6(13.6)$ & $1(2.7)$ & 0.797 & $5.7(0.7-50)$ & \\
\hline Tracheostomy & $3(6.8)$ & $3(8.1)$ & 0.689 & $0.8(0.2-4.4)$ & \\
\hline
\end{tabular}

Abbreviations: MDRAB: Multidrug-resistant Acinetobacter baumannii; SD: Standard deviation; APACHE, Acute physiology and chronic health evaluation; COPD: Chronic obstructive pulmonary disease; *: Apache II score was measured at days admitted to intensive care unit or respiratory care center 
Table 4: The clinical status and their influences on 14-day MDRAB eradiation with aerosolized colistin

\begin{tabular}{|c|c|c|c|c|c|c|}
\hline Variable & $\begin{array}{c}\text { 14-day eradication } \\
n=44(\%)\end{array}$ & $\begin{array}{c}\text { Failed 14-day eradication } \\
n=37(\%)\end{array}$ & $\begin{array}{l}\text { Univariate analysis } \\
\quad p \text { value }\end{array}$ & OR $(95 \% \mathrm{CI})$ & $\begin{array}{l}\text { Multivariate analysis } \\
\qquad p \text { value }\end{array}$ & OR \\
\hline CXR improving & $22(50.0)$ & $15(40.5)$ & 0.395 & $1.5(0.6-3.5)$ & & \\
\hline CXR stationary & $21(47.7)$ & $14(37.8)$ & 0.371 & $1.5(0.6-3.7)$ & & \\
\hline CXR worsening & $1(2.3)$ & $8(21.6)$ & 0.006 & $11.9(1.4-100)^{\S}$ & 0.029 & 12.0 \\
\hline SIRS score improving & $11(25.0)$ & $9(24.3)$ & 0.944 & $1.0(0.4-2.9)$ & & \\
\hline SIRS score stationary & $27(61.4)$ & $17(45.9)$ & 0.165 & $1.9(0.8-4.5)$ & & \\
\hline SIRS score worsening & $6(13.6)$ & $11(29.7)$ & 0.076 & $2.7(0.9-8.1)^{\S}$ & 0.108 & 2.8 \\
\hline Culture-inh days* & $4.0 \pm 2.5$ & $7.3 \pm 6.5$ & 0.002 & & 0.005 & \\
\hline Colonization & $32(72.7)$ & $25(67.6)$ & 0.612 & $1.3(0.5-3.3)$ & & \\
\hline Pneumonia & $12(27.3)$ & $12(36.4)$ & 0.612 & $0.8(0.3-2)$ & & \\
\hline
\end{tabular}

Abbreviations: MDRAB: Multidrug-resistant Acinetobacter baumannii; SIRS: Systemic inflammatory response syndrome; *: Culture-inh days: The interval between the first day that the positive sputum culture for MDRAB was yielded and the first day of the colistin inhalation treatment;

$\dagger$ : OR rate was presented as: failed eradication/success eradication

lower chance of early MDRAB eradication [4/44 (13.6\%) vs. $11 / 37(29.7 \%) ; p=0.076]$. On multivariate analysis, shorter interval between the day that the sputum culture yielded MDRAB positive growth and the day that the aerosolized colistin therapy began $(p=0.005)$, and worsening CXR on Day 7 of the colistin inhalation $(p=0.029)$ still showed a significant lower chance of early MDRAB eradication, but worsening SIRS score on Day 7 was no more a influencing factor of failed MDRAB eradication $(p=0.108)$.

\section{DISCUSSION}

$54.3 \%$ of the patients receiving inhalation colistin for MDRAB had achieved the goal of 14-day eradication. High MDRAB respiratory tract eradication rate using inhalation colistin observed in this study was consistent with previous

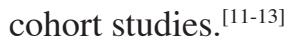

In colistin inhalation group, 27 of 81 patients had concomitant use of IV antibiotics. After 27 patients who with concomitant IV antibiotics were excluded, colistin inhalation group still had higher 14-day eradication than the control group [61.1\% (33/54) vs. $29.6 \%(16 / 38) ; p=0.001]$. Since eradication rate was higher in patients with inhalation alone than in those with combined inhalation with IV antibiotics [61.1\% (33/54) vs. $40.7 \%$ (11/27)], synergistic or additive effect did not seem to exist.

Although aerosolized colistin has been used to eradicate MDRAB from the respiratory tract,,${ }^{[11,13,14,18]}$ we further determined the influencing factors for successful eradication. This retrospective analysis yielded three main findings. First, early intervention using aerosolized colistin in patients with MDRAB-induced pneumonia or colonization can achieve better eradication. Second, patients with DM receiving aerosolized colistin for MDRAB-induced colonization or pneumonia showed worse eradication results. Third, the progression of CXR on Day 7 may predict failed early eradication.
Early prediction of the eradication outcomes of patients with MDRAB colonization or pneumonia enables prompt adjustment of the treatment strategy and a more efficient allocation of medical resources. In addition, early prediction can help control infection and reduce the outbreak of MDRAB. Our study results may be applied to clinical practice because we attempted to use colistin inhalation when the sputum culture grew MDRAB at first and decided whether to continue colistin inhalation on Day 7 after reviewing the CXR change. If the CXR progressed, the inhalation colistin treatment was changed to IV colistin, Tigecycline, or a combined inhaled and IV colistin to enhance MDRAB eradication. ${ }^{[7,15,21-24]}$

We found that patients with early eradication showed a shorter interval between the day that the sputum culture yielded positive MDRAB growth and the day that the aerosolized colistin therapy began. This may be explained as the longer the interval between positive cultures and the beginning of inhalation, the more the bacterial load. In addition, biofilm formation caused by delaying colistin inhalation may make MDRAB eradication more difficult. ${ }^{[25-27]}$

We also investigated how long the colistin inhalation should be used if early eradication was not achieved. In our study, $44(54.3 \%)$ of 81 patients treated with aerosolized colistin had early eradication. However, if early eradication was not achieved, prolonged use of aerosolized colistin benefited an extra $27.0 \%$ (10/37) of patients with 28-day MDRAB eradication. For acute kidney injury, no statistically significant difference was found between patients treated with aerosolized colistin and those treated with other medications [38.1\% (24/63) vs. $30.6 \%(11 / 36) ; p=0.576]$, which is comparable with other study results. ${ }^{[11-13]}$ During the study period, no bronchospasm was found in patients with colistin inhalation, consistent with the results of previous clinical studies. ${ }^{[1-13]}$ Thus, we suggest that for patients who cannot achieve early eradication with a stable clinical status, 
receiving prolonged treatment using aerosolized colistin (for up to 28 days) is reasonable.

We also found patients with DM had lower 14-day eradication rate. Patients with DM are considered to be immunocompromised due to disturbances in cellular innate immunity. ${ }^{[28,29]}$ Furthermore, some microorganisms (e.g., Candida albicans) become more virulent in a high glucose environment. ${ }^{[28,29]}$

A number of limitations should be mentioned when interpreting the results of this study. First, the retrospective design of the study may have omitted factors influencing the eradication outcome, and we did not interfere in the antibiotic treatments used for MDRAB. Second, patients who were included in our analysis consisted of a heterogeneous population with a variety of sources and hospitalization duration before acquiring MDRAB. On the other hand, it can reflect the reality that patients with MDRAB colonization or pneumonia come from heterogeneous populations. Third, patients treated with IV colistin were excluded to avoid the IV treatment influence on MDRAB eradication. In the aerosolized colistin group, $33 \%$ of the patients had concomitant usage of IV antibiotics (22 patients used Tigecycline and 5 patients used Unasyn); in the control group, $74 \%$ patients received IV antibiotics (40 patients received Tigecycline). When considering the influence of the concomitant IV antibiotics, the eradication rate may have been lower in the control group than in the aerosolized colistin group because more patients used IV antibiotics in the control group. However, a recent study showed a comparable eradiation rate between a treatment that combined concomitant aerosolized and IV colistin and the one that consisted solely of IV colistin in patients with ventilator-associated pneumonia. ${ }^{[24]}$ Fourth, there was a high percentage of patients with colonization in colistin inhalation group than in IV antibiotics group [70.4\% (57/81) vs. $48.1 \%$ (26/54); $p=0.009]$. We cannot deny that the higher 14-day eradication rate of MDRAB for colistin inhalation group is due to the difference in severity in both groups since higher colonization percentage accounts for the higher eradication rate. A matched case-control study showed higher 14-day MDRAB eradication rate in colistin inhalation group and control group [84.6\% (33/39) vs. $10.3 \%(4 / 39) ; p<0.001]$ regardless of the percentage of colonization. ${ }^{[1]}$

In conclusion, early intervention for patients with MDRAB pneumonia or colonization using aerosolized colistin may achieve a higher eradication rate. If the CXR progressed on Day 7 of colistin inhalation therapy, early eradication failure may occur.

\section{Acknowledgment}

The authors thank members of the Department of Respiratory Therapy for providing clinical assistance.

\section{REFERENCES}

1. Joshi SG, Litake GM, Niphadkar KB, Ghole VS. Multidrug resistant Acinetobacter baumannii isolates from a teaching hospital. J Infect Chemother 2003;9:187-90.

2. Dijkshoorn L, Nemec A, Seifert H. An increasing threat in hospitals: Multidrug-resistant Acinetobacter baumannii. Nat Rev Microbiol 2007;5:939-51.

3. Perez F, Hujer AM, Hujer KM, Decker BK, Rather PN, Bonomo RA. Global challenge of multidrug-resistant Acinetobacter baumannii. Antimicrob Agents Chemother 2007;51:3471-84.

4. Urban C, Segal-Maurer S, Rahal JJ. Considerations in control and treatment of nosocomial infections due to multidrug-resistant Acinetobacter baumannii. Clin Infect Dis 2003;36:1268-74.

5. Wilson SJ, Knipe CJ, Zieger MJ, Gabehart KM, Goodman JE, Volk HM, et al. Direct costs of multidrug-resistant Acinetobacter baumannii in the burn unit of a public teaching hospital. Am J Infect Control 2004;32:342-4.

6. Tseng CC, Liu SF, Wang CC, Tu ML, Chung YH, Lin MC, et al. Impact of clinical severity index, infective pathogens, and initial empiric antibiotic use on hospital mortality in patients with ventilator-associated pneumonia. Am J Infect Control 2012;40:648-52.

7. Kalin G, Alp E, Coskun R, Demiraslan H, Gundogan K, Doganay M. Use of high-dose IV and aerosolized colistin for the treatment of multidrug-resistant Acinetobacter baumannii ventilator-associated pneumonia: Do we really need this treatment? J Infect Chemother 2012;18:872-7.

8. Doshi NM, Mount KL, Murphy CV. Nephrotoxicity associated with intravenous colistin in critically ill patients. Pharmacotherapy 2011;31:1257-64.

9. Littlewood JM, Miller MG, Ghoneim AT, Ramsden CH. Nebulised colomycin for early pseudomonas colonisation in cystic fibrosis. Lancet 1985;1:865.

10. Valerius NH, Koch C, Hoiby N. Prevention of chronic Pseudomonas aeruginosa colonisation in cystic fibrosis by early treatment. Lancet 1991;338:725-6.

11. Kuo SC, Lee YT, Yang SP, Chen CP, Chen TL, Hsieh SL, et al. Eradication of multidrug-resistant Acinetobacter baumannii from the respiratory tract with inhaled colistin methanesulfonate: A matched case-control study. Clin Microbiol Infect 2012;18:870-6.

12. Perez-Pedrero MJ, Sanchez-Casado M, Rodriguez-Villar S. Nebulized colistin treatment of multi-resistant Acinetobacter baumannii pulmonary infection in critical ill patients. Med Intensiva 2011;35:226-31.

13. Lin CC, Liu TC, Kuo CF, Liu CP, Lee CM. Aerosolized colistin for the treatment of multidrug-resistant Acinetobacter baumannii pneumonia: Experience in a tertiary care hospital in northern Taiwan. J Microbiol Immunol Infect 2010;43:323-31.

14. Michalopoulos A, Kasiakou SK, Mastora Z, Rellos K, Kapaskelis AM, Falagas ME. Aerosolized colistin for the treatment of nosocomial pneumonia due to multidrug-resistant Gram-negative bacteria in patients without cystic fibrosis. Crit Care 2005;9:R53-9.

15. Linden PK, Paterson DL. Parenteral and inhaled colistin for treatment of ventilator-associated pneumonia. Clin Infect Dis 2006;43 Suppl 2:S89-94. 
16. Hartzell JD, Neff R, Ake J, Howard R, Olson S, Paolino K, et al. Nephrotoxicity associated with intravenous colistin (colistimethate sodium) treatment at a tertiary care medical center. Clin Infect Dis 2009;48:1724-8.

17. Koomanachai P, Tiengrim S, Kiratisin P, Thamlikitkul V. Efficacy and safety of colistin (colistimethate sodium) for therapy of infections caused by multidrug-resistant Pseudomonas aeruginosa and Acinetobacter baumannii in Siriraj Hospital, Bangkok, Thailand. Int J Infect Dis 2007;11:402-6.

18. Kwa AL, Loh C, Low JG, Kurup A, Tam VH. Nebulized colistin in the treatment of pneumonia due to multidrug-resistant Acinetobacter baumannii and Pseudomonas aeruginosa. Clin Infect Dis 2005;41:754-7.

19. Michalopoulos A, Fotakis D, Virtzili S, Vletsas C, Raftopoulou S, Mastora Z, et al. Aerosolized colistin as adjunctive treatment of ventilator-associated pneumonia due to multidrug-resistant Gram-negative bacteria: A prospective study. Respir Med 2008;102:407-12.

20. Dellinger RP, Levy MM, Rhodes A, Annane D, Gerlach H, Opal SM, et al. Surviving sepsis campaign: International Guidelines for Management of severe sepsis and septic shock: 2012. Crit Care Med 2013;41:580-637.

21. Shin JA, Chang YS, Kim HJ, Kim SK, Chang J, Ahn CM, et al. Clinical outcomes of tigecycline in the treatment of multidrug-resistant Acinetobacter baumannii infection. Yonsei Med J 2012;53:974-84.

22. Tasbakan MS, Pullukcu H, Sipahi OR, Tasbakan MI, Aydemir S, Bacakoglu F. Is tigecyclin a good choice in the treatment of multidrug-resistant Acinetobacter baumannii pneumonia? J Chemother 2011;23:345-9.

23. Gordon NC, Wareham DW. A review of clinical and microbiological outcomes following treatment of infections involving multidrug-resistant Acinetobacter baumannii with tigecycline. J Antimicrob Chemother 2009;63:775-80.

24. Kofteridis DP, Alexopoulou C, Valachis A, Maraki S, Dimopoulou D, Georgopoulos D, et al. Aerosolized plus intravenous colistin versus intravenous colistin alone for the treatment of ventilator-associated pneumonia: A matched case-control study. Clin Infect Dis 2010;51:1238-44.

25. Rajamohan G, Srinivasan VB, Gebreyes WA. Biocide-tolerant multidrug-resistant Acinetobacter baumannii clinical strains are associated with higher biofilm formation. J Hosp Infect 2009;73:287-9.

26. Lee HW, Koh YM, Kim J, Lee JC, Lee YC, Seol SY, et al. Capacity of multidrug-resistant clinical isolates of Acinetobacter baumannii to form biofilm and adhere to epithelial cell surfaces. Clin Microbiol Infect 2008;14:49-54.

27. Park JY, Kim S, Kim SM, Cha SH, Lim SK, Kim J. Complete genome sequence of multidrug-resistant Acinetobacter baumannii strain 1656-2, which forms sturdy biofilm. J Bacteriol 2011;193:6393-4.

28. Peleg AY, Weerarathna T, McCarthy JS, Davis TM. Common infections in diabetes: Pathogenesis, management and relationship to glycaemic control. Diabetes Metab Res Rev 2007;23:3-13.

29. Vardakas KZ, Siempos II, Falagas ME. Diabetes mellitus as a risk factor for nosocomial pneumonia and associated mortality. Diabet Med 2007;24:1168-71. 